\title{
The Role of Peer Mentors in Promoting Knowledge and Skills Development in Graduate Education
}

\author{
Diane L. Lorenzetti $\mathbb{D}^{1},{ }^{1}$ Lorelli Nowell, ${ }^{2}$ Michele Jacobsen, ${ }^{3}$ Liza Lorenzetti, ${ }^{4}$ \\ Tracey Clancy, ${ }^{2}$ Georgina Freeman, ${ }^{5}$ and Elizabeth Oddone Paolucci ${ }^{6}$ \\ ${ }^{1}$ Health Sciences Library, University of Calgary, 3330 Hospital Drive NW, Calgary, AB, T2N4N1, Canada \\ ${ }^{2}$ Faculty of Nursing, University of Calgary, 2500 University Drive NW, Calgary, AB, T2N1N4, Canada \\ ${ }^{3}$ Werklund School of Education, University of Calgary, 2500 University Drive NW, Calgary, AB, T2N1N4, Canada \\ ${ }^{4}$ Faculty of Social Work, University of Calgary, 2500 University Drive NW, Calgary, AB, T2N1N4, Canada \\ ${ }^{5}$ Department of Medicine, Cumming School of Medicine, University of Calgary, 3280 Hospital Drive NW, Calgary, AB, \\ T2N4Z6, Canada \\ ${ }^{6}$ Department of Community Health Sciences, Cumming School of Medicine, University of Calgary, 3280 Hospital Drive NW, \\ Calgary, AB, T2N4Z6, Canada
}

Correspondence should be addressed to Diane L. Lorenzetti; dllorenz@ucalgary.ca

Received 19 May 2020; Revised 3 October 2020; Accepted 7 October 2020; Published 8 November 2020

Academic Editor: Gwo-Jen Hwang

Copyright (C) 2020 Diane L. Lorenzetti et al. This is an open access article distributed under the Creative Commons Attribution License, which permits unrestricted use, distribution, and reproduction in any medium, provided the original work is properly cited.

\begin{abstract}
The objective of this study was to explore the role of peer mentorship in facilitating graduate student resiliency, knowledge acquisition, and development of academic competencies. We conducted a qualitative case study, using in-person interview data from sixty-two students recruited from four professional faculties (Education, Medicine, Nursing, and Social Work) at a large Canadian University. We identified four broad themes derived from a thematic and constant comparative analysis of interview data: (1) knowledge sharing, (2) skills development, (3) academic milestones, and (4) program supports. Graduate students reported that peer mentorship promoted the development of learning environments that emphasized community, collaboration, and shared purpose. Students believed that peer mentors facilitated their access to essential procedural and disciplinary knowledge and helped them to develop academic and research skills and achieve key academic milestones. While the majority of the students interviewed had not participated in any formal peer-mentoring program, they recommended that any future program incorporate mentorship training and include access to collaborative spaces and targeted opportunities for students to develop these relationships.
\end{abstract}

\section{Introduction}

While international graduate program enrollment continues to increase every year [1], graduate student attrition rates remain a concern across disciplines [2-4]. A number of factors can affect attrition and time to graduation. These include lack of clarity with respect to degree requirements, limited financial support, research topics that are beyond the scope of a thesis, changing family circumstances, competing responsibilities, inadequate supervision, and insufficient writing skills $[5,6]$. Graduate students have reported feeling overwhelmed or confused by their program's degree requirements and lacking in confidence with respect to their research and writing abilities [7]. In a recent qualitative exploration of doctoral students in New Zealand, researchers found that students believed their "preconceptions were at odds with the lived doctoral experience" [7].

Social isolation has also been identified as a key factor contributing to graduate student attrition [8]. In a grounded theory exploration of doctoral students experiences with school-work-life balance, researchers found that lack of support and social isolation were among those 
factors instrumental to doctoral students decisions to withdraw from graduate programs [9]. In a separate study, Ph.D. students acknowledged feeling unprepared "for the degree of loneliness, isolation, and difficulty they would face" [7]. For some students, this sense of isolation can delay degree completion or even result in a failure to graduate $[6,10,11]$. This may be particularly true for international or distance students who find themselves demographically and socially isolated from their cultural or social groups [10].

Peer mentoring can help to minimize graduate student isolation $[11,12]$. In a recent exploration of support systems in higher education, graduate students reported that social support was vital to coping with and overcoming many of the challenges associated with graduate education. [13]. Unlike traditional mentorship, individuals in peer-mentoring relationships are often at similar stages in their education or careers [14]. Hierarchical power differentials are thus reduced, and greater emphasis is placed on mutual learning $[14,15]$. Researchers have suggested that graduate students can derive a variety of psychosocial and even academic benefits from interacting with and learning from one another [16]. For instance, graduate students who have participated in peer writing circles have reported that peer engagement and support promoted increased self-confidence and skills-building $[17,18]$.

While the benefits of peer mentoring for undergraduate students have been established, relatively little is known about the extent to which peer-mentoring relationships can promote knowledge and skills acquisition in graduate education $[19,20]$. The research questions we explored in this study were as follows:

(1) To what extent does participation in informal or formal peer mentoring relationships support knowledge acquisition and the development of academic competencies or skills in graduate education?

(2) How can academic institutions support the development of peer mentoring relationships among graduate students?

\section{Materials and Methods}

We adopted a qualitative case study approach to this exploration of graduate student peer mentoring relationships in a large North American university [21]. Qualitative case study is a methodological framework that supports the conduct of in-depth explorations of discrete events or experiences [21]. We established an a priori definition of peer mentorship as a means of forming a common basis for understanding this complex construct to facilitate communications and conversations between researchers and research participants. Drawing on prior research, we defined peer mentorship as "a reciprocal relationship" characterized by "regular/consistent interaction" between individuals with varying levels of expertise or experience that "produces developmental benefits" valued by mentees in these relationships [22].
2.1. Theoretical Framework. Social learning theories informed the development of the theoretical framework for this study. Social learning theorists posit that knowledge, attitudes, and behaviours develop "through dialogue," collaboration, and cooperation and can be enhanced or cocreated through social interaction [23-26]. Peer mentorship is, above all else, an interactive and collaborative social relationship, typically characterized by sustained interaction between mentors and mentees who share common attributes and personal goals. It is this sustained interaction, or social context, which enables and promotes deep learning [24-26]. These theories informed the development of our interview guide and framed the analysis of student interview data for this study.

2.2. Data Collection. Master's and Ph.D. students currently enrolled in four professional faculties (Education, Medicine, Nursing, and Social Work) in a large Canadian university participated in this study. Faculties selected for inclusion in this study had or were in the process of establishing formal peer-mentorship programs for students in their graduate education programs. Student participants were recruited through listservs, websites, e-newsletters, social media, and word of mouth. Students who had completed one or more years of their graduate programs were invited to participate in this study. Purposeful maximum variation sampling techniques enabled us to explore common and divergent attitudes and experiences of peer mentorship across multiple disciplines. We included students who had and had not as yet participated in formal peer-mentoring programs.

Data were collected through semistructured interviews. The development of the interview guide was informed by a review of the published literature on peer mentorship. The interview guide included questions that encouraged students to reflect on their peer-mentoring relationships (as either mentors or mentees) and the role that peer mentors had played in their academic experiences.

Institutional ethics approval was obtained for this study. Students were asked to review and sign a consent form that outlined the purpose of the study, the nature of participation, and ways in which researchers would ensure the confidentiality of data. Interviews were conducted and digitally recorded by a research assistant and then subsequently transcribed and anonymized. As many of the research team hold faculty positions in the faculties and departments from which students were recruited, designating a research assistant to collect study data was essential to ensure the confidentiality of students' responses.

2.3. Data Analysis. We implemented thematic analysis techniques during our analysis of interview data. Data segments were coded, analyzed, and compared to identify common patterns and points of divergence across data [21]. Open codes (coding labels which mirror students' exact words) were gradually transformed into broader more conceptual themes [27]. Anonymized transcripts were analyzed in duplicate by researchers, and any discrepancies 
were resolved through discussion and consensus. This process of dual analysis strengthened the quality and credibility of the findings derived from our data.

\section{Results}

Sixty-two full-time Master's $(n=28)$ and Ph.D. $(n=34)$ students from four professional faculties (Education E1--E14; Medicine M1--M31; Nursing N1-N7, and Social Work S1-S10) were interviewed for this study. Eighty-two percent $(n=51)$ of these students were women, and $19 \%(n=12)$ self-identified as international students. While all of the students had participated in organic or self-selecting peer-mentoring relationships, $16 \%(n=10)$ of Education students had also been previously matched with peers in a faculty-organized peermentorship program. Our analysis of interview data identified four interconnected broad themes related to the impact of peer mentorship on graduate students' academic experiences: (1) knowledge sharing, (2) skills development, (3) academic milestones, and (4) program supports. We further delineated these themes into nine subthemes (Table 1). Our analyses of subthemes did not reveal any differences in perspectives between students who had and had not participated in formal peermentorship programs.

3.1. Knowledge Sharing. During interviews, graduate students affirmed their belief that knowledge sharing between peers was fundamental to promoting their academic learning. The theme of knowledge sharing was further delineated into three distinct subthemes: (1) navigating the university landscape, (2) exploring diverse perspectives, and (3) accessing peer review and feedback.

3.1.1. Navigating the University Landscape. Students in this study commented on the many "unwritten rules and conventions" (M10) that govern their conduct and progress through academia and the importance of familiarizing themselves with these guidelines. As one student noted, "no matter how many websites you look at or. . . people you talk to, you're not going to find all of the answers that you need to either complete some sort of requirement or some sort of paperwork or some little thing that you were supposed to do" (M3). Most graduate students believed that knowledge shared among peers helped them to access "a much broader and wider knowledge base" (M11) from which to complete their education. Peers promoted academic acclimatization by sharing information on resources, contacts, and processes, including facilities and services, seminars, journal clubs, library services, and "knowledge that you're not going to necessarily get from the calendar...your administrator or your profs" (E6). Students reported that peer mentors also helped them to develop a greater understanding of program expectations, "you don't really know what you're supposed to do...you feel like you're just cruising blindly through grad school," (E14) and
TABLE 1: Impact of peer mentorship (themes).

\begin{tabular}{lc}
\hline Themes & Subthemes \\
Knowledge sharing & Navigating the university landscape \\
& Exploring diverse perspectives \\
& Accessing peer review and feedback \\
\hline Skills development & Developing research skills \\
& Managing relationships \\
& Perfecting career skills \\
\hline & Developing research proposals \\
Academic milestones & Completing candidacy \\
& Advancing degree completion \\
\hline Program supports & - \\
\hline
\end{tabular}

course requirements, "some of these processes aren't very transparent and so it's sometimes a time saver to be able to talk to someone who's gone through that process recently" (M22).

Peers also provided other students with "a sneak peek" (E1) into future milestones in a student's academic journey. As one student noted, peers highlighted "what to expect so it wasn't as dreadful as it seemed at first" (E14), while another commented, "you don't have to scramble around in the dark" (E1). Consulting with peers also gave students a sense of assurance that they were doing what was expected of them in their programs: "I was consulting with them for reassurance. . Oh okay that's what I'm supposed to do, expect or I see that's the level of difficulty" (M8).

3.1.2. Exploring Diverse Perspectives. Students viewed their peer-mentoring relationships as reciprocal, where everyone had ideas and perspectives to share.

We kind of all mentor each other which is helpful ... within a group of people like everyone kind of takes a turn mentoring the other people 'cause we all have slightly different experiences and slightly different skill sets. . .so I think everyone has their own specialty that they're able to contribute to the conversation (M20).

Students noted that one of the benefits of sharing knowledge with peers was having "another perspective that you trust" (M7). Through bouncing "ideas off of one another" (M7), students also developed an appreciation of other ways of approaching and thinking about various issues:

I think the most important thing is to be able to speak to what your [sic] both going through, hear the different sides, hear different perspectives that might give you another way to think about things so that you're not only seeing it from one side (N7).

Students further reported that opportunities to explore different perspectives helped further their understanding of key research concepts and improved their ability to communicate this knowledge to others. One student stressed the following: 
Establishing the collaborative learning environment where participants engaged in the learning share with each other is important. It helps to, to create knowledge, to create a better understanding of the knowledge and to get people accustomed to transmitting that knowledge to others (E11).

3.1.3. Accessing Peer Review and Feedback. Peer review and feedback were viewed as a valuable component of student learning, and an important means by which knowledge was shared among students. One graduate student noted the following:

We started to rely on our peers more ... we could help one another ... just bouncing things off one another you know where is the best place to put this, or what does this look like, or does this sentence make sense you know, and those kind of things help (N3).

Peers provided input and advice on research ideas, ethics applications, abstract and paper development, presentations, research proposals, and scholarship or teaching (teaching assistant) applications. In some instances, peer review was also explicitly incorporated into classroom activities: "it was built in ...so two people would read ... each person's proposal so you would be getting feedback from your peers" (S7).

Finally, peers exemplified a safe space from which to solicit and receive feedback.

There's that level of safety with your peers like I would be less wary about firing off a draft of something to a peer, like having a peer like just look over something for me than I would [be] sending it to someone on my committee (M14).

Outside of classroom environments, students shared that they would often approach their peers for feedback prior to submitting drafts of their work to supervisors or supervisory committees.

3.2. Skills Development. The theme of skills development was prevalent throughout our interview data. Students reported that, through peer mentorship, they were able to develop functional and interpersonal competencies: (1) developing research skills, (2) managing relationships, and (3) perfecting career skills.

\subsubsection{Developing Research Skills. A major component of} graduate studies is the development of those skills necessary to enable students to complete and present their research. Graduate students spoke of the extent to which peers helped them to develop research competencies and knowledge of specific processes including "going to the ethics board for review or developing their research design or their survey questions" (S10) or honing scholarship applications and winning "award[s]" (M31).
Another student also commented on her role in sharing her research expertise with peers:

If people want to know more about participatory action research then I just say don't worry, we'll sit down together. We'll have coffee and then talk about that so that's how I support; I share some of the resources through e-mail for them to read and review and then we sit down together and I talk about participatory action research. I talk about my research, what it looks like, what social justice research looks like (S3).

Students believed their personal mastery of theories and concepts was enhanced through the process of mentoring others: "when you're teaching something to someone else, it forces you to become that much more of an expert on the topic" (E11) and "if I share my knowledge with them, then it will consolidate my knowledge foundation" (M23).

An important aspect of this type of peer mentorship was encouraging those who had received peer support to share their newfound skills with others: "We celebrate each other's success genuinely. We celebrate them and then we bring them back to be a part of that panel that then gives advice to the rest of the group on how they can be successful" (M8). In all faculties, formal scholarship preparation meetings provided students with opportunities to "ask [senior peers] what grants they applied [for] and how they set up their research proposals" (M20). Students who had access to prior successful scholarship applications found it "really helpful just to see how, how to apply and how to do things" (E14). As one student confirmed, "I've been quite successful in getting scholarships and I attribute a lot of that to the mentorship that I've received" (M30).

While graduate students are generally expected to submit abstracts and present papers at local, national, and international conferences, some shared that they "had no idea what a poster should look like, what these presentations at conferences should look like" (M14). For these students, the opportunity to "present in front of one another" and receive nuanced feedback from peers "was really helpful" (N3). Others went further, commenting that their ability to present research at conferences was due, in part, to having access to peer input and advice on abstract submissions.

One of the posters that I submitted was only accepted because one of my peer mentors actually reviewed it, read it, and was like no, start again. It was completely different the second time around and it would not have been accepted because it was nonsense the first one (M11).

While academic writing is a key component of graduate school, for many students "scholarly writing does [not] come natural" (N3). Some students acknowledged that they felt insecure with respect to their writing expertise, "[we] just think we can't write well...you question yourself...every time you hand in a paper. . .am I doing this right?" (N2), and credited writing groups or circles with improving the quality of their writing: 
We would often correct each other's papers or provide feedback. In the beginning we all had been out of school for a long time... our first papers were terrible (laughter) ... it really is awesome how [my writing has] come along and over time (N1).

As another student confirmed, "when you edit someone else's paper, you sometimes catch those things that you didn't necessarily recognize [in] your own [writing]... when you start recognizing it in someone else's paper, you go oh did I do that in mine?" (N2).

3.2.2. Managing Relationships. While students in this study relied on their supervisors and supervisory committees to guide their progress towards degree completion, some noted these relationships could be challenging. They sometimes experienced "bumps in the road" and "some tussles" (N1) with their supervisors and committee members, leaving them uncertain as to how to navigate these important relationships. One student commented

The graduate supervisor really does kind of hold your success in their hands. If the relationship isn't going well, I think people just feel so awful about themselves and they feel alone. They just feel like they have nowhere to turn (S7).

Students in this study shared they were often hesitant to air their concerns with supervisors or other faculty members, preferring to approach peers who had experienced "similar situation[s]" (M26) and could provide guidance or "advice on how to handle these relationships" (M19). One student explained, "We've [peer-mentorship group] had students that have been really struggling with their supervisors and we've walked them through it and provided them with ideas and support" (N5).

Many graduate students in this study viewed peermentoring relationships as essential to enabling them to begin to develop their professional networks. They spoke of the role that peers play in facilitating access to future job opportunities and the importance of identifying colleagues who could participate in both current and future research collaborations.

I'm investing in my future academic relationships. Out of all the people here that I've worked with, I've seen their work and I've seen what they can do. Here's someone I want to collaborate with on a research project going forward. Here's someone I definitely never want to work with again. I'm building all my networks for when I'm leading my own research projects and I know who I'm going to work with and I know who I'm never going to work with (M30).

Finally, students recognized that mentoring others helped them to develop interpersonal skills that would be transferable across career trajectories: "it's just good career practice because you will always have to mentor someone or you'll have to manage someone or you just need to learn those interpersonal skills" (M19). Another student confirmed that "being a mentor helps to lay the foundation for being a good supervisor, a good teacher" (E1).

3.2.3. Perfecting Career Skills. Career skills were defined as key organizational competencies, including time management and personal and professional accountability. Peers were seen as essential to promoting personal accountability and providing advice on time management. Some students met regularly in groups as a way of ensuring "accountability about writing" (N6) and to "hold each other accountable to both school [and] health-related milestone[s]" (N7). One student specifically commented that her peer mentors helped her to balance the competing demands associated with her degree, and develop important time management skills:

Through peer mentorship, it became easier to identify what I needed and how to go about those things just through the discussions that we had. All of us were trying to balance different things in our lives and so yeah it became really easy to identify like this is what I need and this is how much time I need for this on a given day, week, month or whatever (S6).

Students also noted that being involved in peer mentorship helped them to develop academic self-confidence and reflective practice, and cultivate the skills and willingness to help others. One respondent mused:

I think every time you support someone else no matter what it is, there is some personal benefit to you. One, it refines your skills in terms of your coaching and mentoring skills and two, it helps you to reflect on your own needs in terms of having a mentor in your life as well but also it helps you to reflect on your knowledge and skills in that particular area (S10).

3.3. Academic Milestones. Finally, graduate students provided examples of how peer-mentoring relationships enabled them to further key academic milestones, including (1) developing research proposals, (2) completing candidacy, and (3) advancing degree completion.

3.3.1. Developing Research Proposals. In Canada, thesisbased graduate students (Master's and Ph.D.) are required to complete a research proposal as part of their degree progression. Most of the students we interviewed stated that they had informally reached out to peers for advice on structuring their research proposals, while others shared that they had participated in structured peer support initiatives offered through their programs: "they assign you know a senior, a Ph.D. student to mentor us how to write the proposal. . .she helped me to improve my research proposal" (M23). Some students shared a preference for approaching peers rather than supervisors for advice on developing their proposals and determining their "research directions" (E6). 
This preference was framed as a reluctance to appear less than knowledgeable to supervisors: "no one wants to ask their supervisor how [to] write a proposal but you can ask your friends" (M25).

3.3.2. Completing Candidacy. Candidacy exams constitute a major program component and milestone for $\mathrm{Ph}$. D. students, with many students owning that the "mystique about candidacy" (N6) can cause both uncertainty and stress. As one student explained:

The pieces around process especially around candidacy were quite confusing because there isn't uniformity across the departments and across the school. It seems that everybody experiences something really different. It's quite stressful so I would say that figuring out the logistics around that was challenging (M30).

Ph.D. students often looked to peers who had already been through this milestone for greater clarity on the process.

When I was heading up towards candidacy, he [peer] said you know ... if you can just get through it you'll be part of the club. I've been through it, it's super tough, [but] you're going to get through it too and it's going to be okay. So just that sort of reassurance of someone who knows you, who's got your back and is able to point towards the pieces that are worth worrying about and the pieces that are not worth worrying about. . that was super helpful (M30).

While some students met individually, others organized group meetings to discuss and prepare for completing this stage of their degrees: "A lot of students met with me and did a mock candidacy exam or a mock defense that was very helpful" (M8).

Although peer support was viewed as important, students also noted the timing of this support factored into its impact:

They brought in a student to talk about her candidacy experiences, but at that time we were still in the first year of taking classes. I wasn't thinking of candidacy at that point. It was a bit too early and I think everybody felt the same way as we were still trying to get through classes (S10).

3.3.3. Advancing Degree Completion. While none of the students who were interviewed had completed their studies, many noted that the support they received from peers helped them to remain "on track and involved and motivated" (M14) about their programs. One student explained, "You're going to need someone sometimes to help tell you that no, no you're not quitting, you're not allowed to...that's important and sometimes you're the person who gives that advice" (E10). Student participants appreciated "opportunities to communicate with [students] one or two steps ahead of [them] in the journey" (E12) and believed they would not be on the same "academic or professional development trajectory" (E12) without their peers. Finally, as students neared degree completion and began to plan their career options, some looked to peers who had already graduated to share "the opportunities after graduation [and help them identify and] consider [career] options" (M1).

3.4. Program Supports. Students were asked to consider what program or institution-level supports might have furthered their ability to develop peer-mentoring relationships. Although a few students $(n=10 ; 16 \%)$ had benefited from participation in a formal peer-mentorship program, many shared their preferences with respect to the development and implementation of future structured programs. Students' responses focused on the importance of promoting the value of peer mentorship, mentorship training, relationship building, and the need for space. Suggested strategies ranged from public recognition of "students who are willing to step up as mentors" (M31) to creating learning environments where peer mentorship is viewed as integral to the graduate education experience. One student shared the following:

When I needed help from someone, it was really easy for me to approach [them]. . because there was that sort of environment [where] it was oh yeah of course you do n't know everything so we have to rely on each other (S6).

Those who had mentored other students believed there was also a role for formal programs in facilitating the development of relational skills to better enable students to manage their peer-mentorship roles. Students felt adequate preparation was essential to ensuring positive peer-mentoring experiences for everyone: "the training piece could be a little bit more focused on practical aspects how to manage that relationship" (E2).

Students across disciplines spoke of the importance of faculties and departments "creating those opportunities for those informal interactions" (M2). Suggestions focused on creating "lists of people who are willing to" (M8) act as peer mentors; and organizing "specific activities... where [students could] get together" (S9) to "talk about their experiences" (E8). A few students commented that the sustainability of structured initiatives would be assured if they were "structured somehow around the academic day so that it fits within the frame of what is already going on" (M11).

Feedback also centered on the absence of space, with many students commenting that programs lacked collaborative work rooms or other designated spaces where graduate students could meet to share, discuss, and collaborate. Students reported that available space was integral to the development and sustainability of these relationships: "the physical space is an interesting quandary. If you can't offer your students a place to congregate. . then maybe it is more difficult to establish some of these relationships" (M7). Such space was also seen as essential to ensure the privacy required to discuss sensitive issues or concerns: “There's just no space for anyone anywhere. It is really hard. . to have those conversations in a somewhat private safe space rather than doing it in the middle of the grad lounge where everyone's door is open" (N4). 


\section{Discussion}

This study addresses an important gap in the mentoring literature by providing greater understanding of the impact of peer mentorship on graduate students' academic experiences. While prior studies of peer mentorship in graduate education have primarily focused on the peer mentoring experiences of students in single disciplines $[7,17,18,20,33-36]$, we have highlighted those commonalities of experience and need that exist across disciplines.

Peer mentoring is depicted as a multifaceted relationship that can impact all stages of graduate education; it can promote skills building and the development of key research competencies, engender a sense of accountability and motivation, and encourage and enable students to persist with the completion of major academic milestones. Communities of practice are collectives of individuals who "share experiences and knowledge [with one another] in free-flowing ways that foster new approaches to" solving problems and achieving common goals [28]. Our study found that graduate students who connect with one another early on in their degrees may self-organize into ongoing collaborative peer groups or communities of practice that create a framework for engagement in mutual learning and the provision of psychosocial and practical supports. Academic institutions can encourage and participate in this process by creating academic cultures that celebrate the role of peers in the academic experience and providing students with the training and space to facilitate the development of these relationships.

The development of transferable professional and research skills is fundamental to graduate student learning [29]. These skills can be enhanced through communication, collaboration, and exploring the beliefs, experiences, and perspectives of others [30]. The traditional model of graduate education positions supervisors, supervisory committees, and other faculty members as primary sources of information, guidance, and assistance for students. While faculty members provide essential guidance, oversight, and expertise to enable students to complete their degrees, peers appear to contribute a complementary level of ongoing support that is distinct from that which students receive from supervisors or other academic faculty. In common with other published research, the findings from this study indicate that the immediacy and commonality of experience peers, even near-peers, share can create a trust and a willingness to learn from and rely on one another [31, 32]. Peer relationships may help to address motivational gaps, encouraging and enabling some students to continue to pursue their education. The breadth and significance of these relationships, while relationally distinct, may be as consequential as those that develop with academic supervisors and supervisory committees.

This study is also unique in its exploration of the role institutions can play in promoting the development of peermentoring relationships in graduate education. Central to this is the importance of creating academic cultures where peer mentorship is valued and encouraged. Although many students can and do organically self-select into peer- mentoring relationships, not all may have equal access to these relationships [37]. In particular, those who are culturally, racially, geographically, or otherwise isolated from their peers may find it difficult to identify effective peer mentors [37]. Time and distance can also impinge on equitable access to peer mentorship. Hence, the potential value of formal programs designed to provide equitable access to peer mentoring for graduate students, including international and distance students, who may experience additional challenges in forming supportive peer relationships.

Increasingly, academic institutions appear to be channeling resources towards the development of formal peermentorship programs, primarily for undergraduate students, but also those in higher-degree programs $[7,17,18,20,33-36]$. These initiatives strive to create environments that encourage mutual learning, the sharing of expertise among peers, and the provision of psychosocial, and practical supports. This exploration of the graduate student peer-mentoring experience has uncovered data that can inform the design of future peer-mentoring programs, in particular, those program-level factors that may ensure the wide-scale acceptance and impact of these initiatives.

Our study has caveats and limitations. All of the students who participated in this study were enrolled in one Canadian academic institution. While our multidisciplinary population mix and mixed methods research approach enabled us to capture a wide variety of graduate student experiences and perspectives across four professional disciplines of study, the common organizational culture in which all students were situated may have influenced the resultant themes. The inclusion of students from other academic institutions may have uncovered other, perhaps contrary, perspectives than those presented here. Future research is needed to determine the transferability of these findings to other disciplines and academic institutions. Such efforts should focus on implementing and evaluating the impact that disciplinary and institution-level efforts can have on promoting the development of these relationships and assessing the transferability of study findings to other disciplines and academic institutions. Data from student exit interviews could also help to confirm the role of peer support in graduate student attrition. While we did not interview students who had graduated or withdrawn from their programs, future research in the form of student exit interviews would help to confirm the strength of the impact that peer mentors have on degree completion or decisions to withdraw from graduate programs. Further, this data would enable institutions and program coordinators determine if and how to resource initiatives focused on supporting the development of these relationships.

\section{Conclusions}

Degree persistence and completion in graduate education remains a concern for educational institutions worldwide. This research demonstrates that peer-mentoring relationships can positively affect knowledge acquisition, skills development, and support degree persistence. While many students organically form peer relationships early in their 
degrees, institutional strategies aimed at supporting the development and sustainability of peer mentoring may be essential to increase students' ability to access and benefit from these relationships. The findings from this study suggest that institutional strategies incorporate both structured opportunities for graduate students to interact and physical spaces to encourage the ongoing development of meaningful peer mentoring relationships.

\section{Data Availability}

The data that support the findings of this study are not publicly available due to the inclusion of information that could compromise the privacy of research participants.

\section{Conflicts of Interest}

The authors declare no conflicts of interest.

\section{Acknowledgments}

This work was supported by the University of Calgary Teaching and Learning grant and a grant from the Office of Health \& Medical Education Scholarship (University of Calgary).

\section{References}

[1] OECD, Education at a Glance 2016, OECD Publishing, Paris, France, 2016.

[2] Canadian Association for Graduate Studies, 41st Statistical Report, Canadian Association for Graduate Studies, Ottawa, Canada, 2015.

[3] Council of Graduate Schools, Graduate Enrollment and Degrees: 2003 to 2013, CGS, Washington, DC, USA, 2014.

[4] H. Okahana and E. Zhou, Graduate Enrollment and Degrees 2006 to 2016, Council of Graduate Studies, Washington, DC, USA, 2017.

[5] S. K. Gardner, "Student and faculty attributions of attrition in high and low-completing doctoral programs in the United States," Higher Education, vol. 58, no. 1, pp. 97-112, 2009.

[6] R. G. Ehrenberg, G. H. Jakubson, J. A. Groen, E. So, and J. Price, "Inside the black box of doctoral education: what program characteristics influence doctoral students' attrition and graduation probabilities?" Educational Evaluation and Policy Analysis, vol. 29, no. 2, pp. 134-150, 2007.

[7] E. M. Johnson, "Toward the building of a cross-disciplinary doctoral research and writing culture," Journal of University Teaching and Learning Practice, vol. 11, no. 1, 2014.

[8] D. Jairam and H. D. Kahl Jr., "Navigating the doctoral experience: the role of social support in successful degree completion," International Journal of Doctoral Studies, vol. 7 , pp. 311-329, 2012.

[9] E. Martinez, C. Ordu, M. R. D. Sala, and A. McFarlane, "Striving to obtain a school-work-life balance: the full-time doctoral student," International Journal of Doctoral Studies, vol. 8, pp. 39-59, 2013.

[10] J. L. Lott II, S. Gardner, and D. A. Powers, "Doctoral student attrition in the STEM fields: an exploratory event history analysis," Journal of College Student Retention: Research, Theory \& Practice, vol. 11, no. 2, pp. 247-266, 2009.

[11] S. K. Gardner, "Contrasting the socialization experiences of doctoral students in high-and low-completing departments: a qualitative analysis of disciplinary contexts at one institution," The Journal of Higher Education, vol. 81, no. 1, pp. 61-81, 2010.

[12] J. Stubb, K. Pyhältö, and K. Lonka, "Balancing between inspiration and exhaustion: Ph.D. students' experienced sociopsychological well-being," Studies in Continuing Education, vol. 33, no. 1, pp. 33-50, 2011.

[13] V. T. Byers, R. N. Smith, E. Hwang et al., "Survival strategies: doctoral students' perceptions of challenges and coping methods," Internaltional Journal of Doctoral Studies, vol. 9, pp. 109-136, 2014.

[14] K. E. Kram and L. A. Isabella, "Mentoring alternatives: the role of peer relationships in career development," Academy of Management Journal, vol. 28, no. 1, pp. 110-132, 1985.

[15] J. L. Murdock, N. Stipanovic, and K. Lucas, "Fostering connections between graduate students and strengthening professional identity through co-mentoring," British Journal of Guidance \& Counselling, vol. 41, no. 5, pp. 487-503, 2013.

[16] B. Holmes, M. Trimble, and D. Morrison-Danner, "Advancing scholarship, team building, and collaboration in a hybrid doctoral program in educational leadership," Journal of College Teaching \& Learning (TLC), vol. 11, no. 4, pp. 175-180, 2014.

[17] C. Guerin, V. Xafis, D. V. Doda et al., "Diversity in collaborative research communities: a multicultural, multidisciplinary thesis writing group in public health," Studies in Continuing Education, vol. 35, no. 1, pp. 65-81, 2013.

[18] D. Cuthbert and C. Spark, "Getting a GRiP: examining the outcomes of a pilot program to support graduate research students in writing for publication," Studies in Higher Education, vol. 33, no. 1, pp. 77-88, 2008.

[19] A. D. Sprengel and L. Job, "Reducing student anxiety by using clinical peer mentoring with beginning nursing students," Nurse Educator, vol. 29, no. 6, pp. 246-250, 2004.

[20] A. Chester, L. J. Burton, S. Xenos, and K. Elgar, "Peer mentoring: supporting successful transition for first year undergraduate psychology students," Australian Journal of Psychology, vol. 65, no. 1, pp. 30-37, 2013.

[21] S. B. Merriam, Qualitative Research: A Guide to Design and Implementation, Jossey-Bass, San Francisco, CA, USA, 2009.

[22] D. L. Haggard, T. W. Dougherty, D. B. Turban, and J. E. Wilbanks, "Who is a mentor? A review of evolving definitions and implications for research," Journal of Management, vol. 37, no. 1, pp. 280-304, 2011.

[23] S. B. Merriam, R. S. Caffarella, and L. M. Baumgartner, Learning in Adulthood: A Comprehensive Guide, John Wiley \& Sons, San Francisco, CA, USA, 2007.

[24] E. C. Wenger and W. M. Snyder, "Communities of practice: the organizational frontier," Harvard Business Review, vol. 78, no. 1, pp. 139-145, 2000.

[25] J. Lave and E. Wenger, Situated Learning: Legitimate Peripheral Participation, Cambridge University Press, Cambridge, UK, 1990.

[26] A. Bandura, "Self-efficacy: toward a unifying theory of behavioral change," Psychological Review, vol. 84, no. 2, pp. 191-215, 1977.

[27] M. B. Miles, A. M. Huberman, and J. Saldaña, Qualitative Data Analysis: A Methods Sourcebook, SAGE, Thousand Oaks, CA, USA, 3rd edition, 2014.

[28] C. L. Tax, H. Doucette, N. R. Neish, and J. P. Maillet, "A model for cultivating dental hygiene faculty development within a community of practice," Journal of Dental Education, vol. 76, no. 3, pp. 311-321, 2012. 
[29] M. Rose, Graduate Student Professional Development: A Survey with Recommendations, Canadian Association for Graduate Studies, Ottawa, Canada, 2012.

[30] D. A. Kolb, Experiential Learning: Experience as the Source of Learning and Development, Prentice-Hall, Englewood Cliffs, NJ, USA, 1984.

[31] A. Macoun and D. Miller, "Surviving (thriving) in academia: feminist support networks and women ECRs," Journal of Gender Studies, vol. 23, no. 3, pp. 287-301, 2014.

[32] L. S. Giddings and P. J. Wood, "How to survive (and enjoy) doing a thesis: the experiences of a methodological working group," Nursing Praxis in New Zealand, vol. 22, no. 1, pp. 11-22, 2006.

[33] M. Snowden and T. Hardy, "Peer mentorship and positive effects on student mentor and mentee retention and academic success," Widening Participation \& Lifelong Learning, vol. 14, pp. 76-92, 2012.

[34] R. Parker, "A learning community approach to doctoral education in the social sciences," Teaching in Higher Education, vol. 14, no. 1, pp. 43-54, 2009.

[35] C. Fleck and M. E. Mullins, "Evaluating a psychology graduate student peer mentoring program," Mentoring \& Tutoring: Partnership in Learning, vol. 20, no. 2, pp. 271-290, 2012.

[36] M. A. Alonso, G. Castaño, A. M. Calles, and S. SánchezHerrero, "Assessment of the efficacy of a peer mentoring program in a university setting," The Spanish Journal of Psychology, vol. 13, no. 2, pp. 685-696, 2010.

[37] N. Haskins, M. Whitfield-Williams, M. A. Shillingford, A. Singh, R. Moxley, and C. Ofauni, "The experiences of black master's counseling students: a phenomenological inquiry," Counselor Education and Supervision, vol. 52, no. 3, pp. 162-178, 2013. 